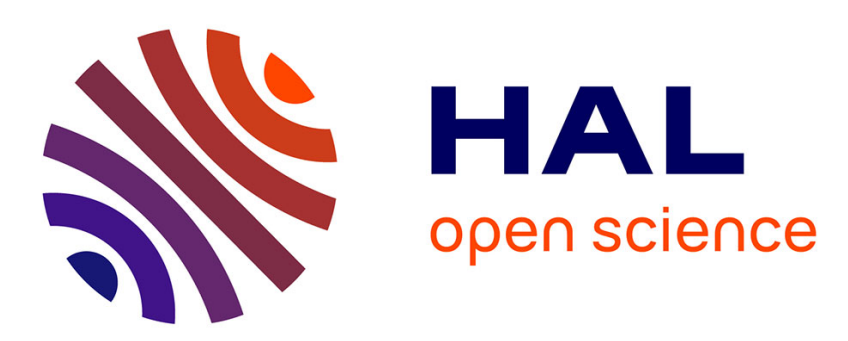

\title{
Physics-based oligomeric models of the yeast mitofusin Fzo1 at the molecular scale in the context of membrane docking
}

Astrid Brandner, Dario de Vecchis, Marc Baaden, Mickaël M Cohen, Antoine Taly

\section{To cite this version:}

Astrid Brandner, Dario de Vecchis, Marc Baaden, Mickaël M Cohen, Antoine Taly. Physics-based oligomeric models of the yeast mitofusin Fzo1 at the molecular scale in the context of membrane docking. Mitochondrion, 2019, 49, pp.234-244. 10.1016/j.mito.2019.06.010 . hal-02345043

\section{HAL Id: hal-02345043 \\ https://hal.science/hal-02345043}

Submitted on 1 Dec 2020

HAL is a multi-disciplinary open access archive for the deposit and dissemination of scientific research documents, whether they are published or not. The documents may come from teaching and research institutions in France or abroad, or from public or private research centers.
L'archive ouverte pluridisciplinaire HAL, est destinée au dépôt et à la diffusion de documents scientifiques de niveau recherche, publiés ou non, émanant des établissements d'enseignement et de recherche français ou étrangers, des laboratoires publics ou privés. 


\section{Title}

6 Authors:

7 Astrid Brandner ${ }^{1}$, Dario De Vecchis ${ }^{1}{ }^{*}$, Marc Baaden $^{1}$, Mickael M. Cohen ${ }^{2 \#}$ and Antoine Taly ${ }^{1 \#}$

$9 *$ Contributed equally

10 " Correspondance

\section{Physics-based oligomeric models of the yeast mitofusin Fzo1 at molecular-} scale in the context of membrane docking.

\section{Affiliations:}

Institut de Biologie Physico-Chimique, Centre National de la Recherche Scientifique, Paris, France. ${ }^{1}$ Laboratoire de Biochimie Théorique, UPR 9080.

${ }^{2}$ Laboratoire de Biologie Cellulaire et Moléculaire des Eucaryotes, Sorbonne Université, CNRS, UMR 8226.

*Correspondence to: $\underline{\text { cohen@ibpc.fr }}$ or taly@ibpc.fr 
ABSTRACT

Tethering and homotypic fusion of mitochondrial outer membranes is mediated by large GTPases of the Dynamin-Related Proteins family called the mitofusins. The yeast mitofusin Fzo1 forms high molecular weight complexes and its assembly during membrane fusion likely involves the formation of high order complexes. Consistent with this possibility, mitofusins form oligomers in both cis (on the same lipid bilayer) and trans to mediate membrane attachment and fusion.

Here, we rely on our recent Fzo1 model to investigate and discuss the formation of cis and trans mitofusin oligomers. We have built 3 cis-assembly Fzo1 models that gave rise to 3 distinct transoligomeric models of mitofusin constructs. Each model involves two main components of mitofusin oligomerization: the GTPase and the trunk domains. The oligomeric models proposed in this study were further assessed for stability and dynamics in a membrane environment using a coarse-grained molecular dynamics (MD) simulation approach. A narrow opening 'head-to-head' cisoligomerization (via the GTPase domain) followed by the antiparallel 'back-to-back' transassociations (via the trunk domain) appears to be in agreement with all the available experimental data. More broadly, this study opens new possibilities to start exploring cis and trans conformations for Fzo1 and mitofusins in general but also for other fusion-DRPs. 


\section{INTRODUCTION}

Mitochondria are dynamic organelles organized as a cytoplasmic reticulum. Mitochondria fuse their outer and inner membranes to form tubules. These mitochondrial tubules can interconnect through fusion but can also fragment through fission of their membranes to yield a network with remarkable plasticity. Together, fusion and fission thus regulate the whole morphology and dynamics of the mitochondrial network, which makes these processes essential for maintenance of mitochondrial integrity and consequently all mitochondrial functions (Friedman and Nunnari, 2014; Shutt and McBride, 2013; Tilokani et al., 2018; Westermann, 2010).

From a mechanistic point of view, mitochondrial fission as well as fusion of outer and inner membranes are all mediated by members of the Dynamin-Related Proteins family (Ramachandran, 2018). These large GTPases act as remodelers of intracellular lipid bilayers through two properties: their capacity to bind biological membranes and their propensity to oligomerize into high order macromolecular structures. Consistent with these features, fission DRPs are recruited to mitochondrial outer membranes by specific adaptor proteins, where they auto-oligomerize upon binding of GTP to assemble into macromolecular spirals that wrap around mitochondrial tubules (Tilokani et al., 2018). Subsequent GTP hydrolysis induces conformational rearrangements of the dynamins, which result in reduced diameter of the spirals and constriction of mitochondrial tubules, followed by their separation.

Fusion-DRPs, on the other hand, are transmembrane proteins that promote homotypic merging of the lipid bilayers in which they are inserted (Cohen and Tareste, 2018). The mitofusins Mfn1 and Mfn2 (Fzo1 in yeast) fuse mitochondrial outer membranes whereas fusion between inner membranes is mediated by Opal (Mgm1 in yeast). Another key member of fusion DRPs is the Atlastin ATL-1 (Sey1 in yeast) that merges membranes from ER tubules. Mitofusins and Atlastins have in common to auto-oligomerize in trans (from opposing lipid bilayers) and in a GTP binding and hydrolysis-dependent manner to tether opposing lipid bilayers and promote their fusion (Cohen and Tareste, 2018). Recent crystal structures of protein portions lacking the TM regions of ATL-1, Sey1 and MFN1 further allow depicting the assembly of DRPs during membrane fusion as protein dimers interacting in trans through their respective GTPase domains (Bian et al., 2011; Byrnes et al., 2013; Cao et al., 2017; Moss et al., 2011; Yan et al., 2018; Yan et al., 2015). However, the yeast mitofusin Fzo1 also forms high molecular weight complexes (Rapaport et al., 1998). In particular, Fzo1 was shown to trigger the formation of a ring-shaped macromolecular complex during mitochondrial docking (Brandt et al., 2016), suggesting that DRPs assembly during membrane fusion might involve the formation of high order complexes rather than solely trans homodimers.

Consistent with this possibility, mitofusins form oligomers in both cis (on the same lipid bilayer) and trans to mediate membrane attachment and fusion (Santel et al., 2003)(Ishihara et al., 2004; Griffin and Chan, 2006; Cao et al., 2017; Koshiba et al., 2004; Anton et al, 2011; Shutt et al., 2012). Besides the GTPase domain, mitofusins also include two heptad-repeat domains (HR1 and HR2) that may get involved in homotypic interactions during mitochondrial tethering (Koshiba et al., 2004; Griffin and Chan, 2006; De Vecchis et al., 2017). Whether GTPase and HR domain interactions take place in cis or trans before or during mitochondrial tethering remains to be investigated or confirmed (Franco et al., 2016; Koshiba et al., 2004).

Here, we rely on our recent Fzo1 model (De Vecchis et al., 2017) to investigate and discuss distinct hypotheses for the formation of cis and trans mitofusin oligomers. Besides being the only near full-length model as of today, it contains a membrane domain, which is a requisite for investigating mitofusin orientations in a bilayer. We employed a modelling procedure that has been guided by available experimental data from the literature. The models proposed in this study were further assessed for stability and dynamics in a membrane environment using a coarse-grained molecular dynamics (MD) simulation approach. This study opens new possibilities to start exploring cis and trans conformations for Fzo1 and mitofusins in general but also for other fusionDRPs. 


\section{METHODS}

The modelling work presented here builds upon an experimentally validated model of the monomeric unit, namely our previously published model of Fzo1 in a closed conformation (De Vecchis et al., 2017). It is first used to generate a monomeric model of the open conformation. Those two models are the basis for the construction of Fzo1 dimer models in cis and then tetramers, via the dimerization in trans of Fzo1 cis-dimers.

\section{Modelling the Fzo1 GTPase dimer construct}

Two chains of the Fzo1 model in closed conformation (De Vecchis et al., 2017) were placed by superimposing their GTPase domains onto that of BDLP in open conformation (PDB-id 2W6D, Low et al., 2009). Then, only the coordinates of the two fragments that comprise the Fzo1 GTPase domain (res 188-461) were retained to form the final GTPase domain dimer model. The loop refinement tool implemented in MODELLER (Fiser et al., 2000) was used to remove a clash in both chains involving an unresolved loop in the template 2J68 (Low and Löwe, 2006) (res 215-219). Models were ranked according to the discrete optimized protein energy (DOPE) method (Shen and Sali, 2006), selecting the best-scoring loop out of 20 candidates.

\section{Modelling cis-dimer configurations}

108

The Fzo1 head-to-head cis-dimer. Two Fzol chains in closed conformation (De Vecchis et al., 2017) were oriented facing each other within a compatible distance to accommodate two interacting GTPase domains. Subsequently, the coordinates of residues 188-440 enclosed between hinges 2a, $2 b$ (i.e. comprising the GTPase domain) were removed from both chains and replaced with the GTPase dimer construct described above. The latter was manually positioned between the two deleted chains resulting in the head-to-head interaction dimer (Fig. 2b). The backbone interruptions were connected using the loop refinement tool implemented in MODELLER (Fiser et al., 2000) using positions 185,188 and 436,445 as anchors. Solutions were ranked according to the DOPE method (Shen and Sali, 2006), selecting the best-scoring loop out of 20 candidates.

The Fzo1 back-to-back cis-dimer. Two chains of the Fzo1 model in closed conformation (De Vecchis et al., 2017) were manually oriented with respect to each other in order to generate the back-to-back interaction (discussed in the text). In the resulting model system (Fig. 2c) the HR domains face each other in a parallel fashion.

The Fzo1 open cis-dimer. The coordinates from BDLP in open conformation (PDB-id 2W6D, Low et al., 2009) derived from the electron density map of native BDLP lipid tubes (accession code: EMD-1589) were used as template to model Fzo1 in open conformation. Starting from our previous Fzo1-BDLP target-template alignment (De Vecchis et al., 2017) and using an analogous approach to the one described in Low et al., 2009, we introduced homologous chain breaks on the Fzo1 model (De Vecchis et al., 2017), resulting in five rigid blocks (Fig. 1a). Each fragment was superposed to its corresponding fragment in $2 \mathrm{~W} 6 \mathrm{D}$, in order to reconstitute the orientation found in BDLP (Fig. 1). The MatchMaker tool from the UCSF Chimera software (Pettersen et al., 2004) was used during this procedure. The loop refinement tool implemented in MODELLER (Fiser et al., 2000) enabled us to complete the model in the resulting backbone interruptions and to remove a clash in both chains between the side chain of the Lys 271 and the backbone of the Ala401 residues, using positions 268 and 273 as anchors. Solutions were ranked according to the discrete optimized protein energy (DOPE) method (Shen and Sali, 2006), selecting the best-scoring loop out of 10 models.

\section{Modelling the trans-dimer configurations}


The Fzo1 head-to-head trans tethered tetramer in open conformation. Two Fzo1 open cis-dimer models obtained as described above were manually oriented to mimic the interactions in trans towards their respective GTPase domains. In the resulting model system the two transmembrane segments are located at opposite ends (Fig. 5a).

The Fzol head-to-head trans tethered tetramer (antiparallel). The transmembrane segment of two Fzo1 head-to-head cis-dimers described above were manually oriented at opposite ends in order to optimize the interaction between their respective HR domains oriented in an antiparallel fashion (Fig. 5c). Note that this system, although antiparallel, could also be considered as back-to-back.

The Fzo1 head-to-head trans tethered tetramer (parallel). Two Fzo1 back-to-back cis-dimers obtained as described above were initially positioned with the respective transmembrane segments at opposite ends in order to mimic the supposed tethering process. Subsequently, the coordinates of residues 101-491 and 816-855 enclosed between hinges 1a, 1b (i.e. comprising the GTPase domain and the 3-helix bundle), were removed from the two resulting juxtaposing chains. Then, in a similar way the GTPase dimer construct was built (see above), we superposed the GTPase domain alpha carbons of two Fzo1 chains in closed conformation (De Vecchis et al., 2017) with the human mitofusin dimer (PDB-Id: 5GOM, Cao et al., 2017). This choice was motivated and directly inspired by the work from Gao and collaborators that proposed a possible Mfn1 trans cross oligomer (Cao et al., 2017). From the resulting Fzo1 dimer, only the GTPase domain and the 3helix bundle were selected and used to replace the aforementioned deleted portions, then generating the trans head-to-head interaction (Fig. 5b). A clash in one chain (res 215-218) was removed from the resulting Fzo1 dimer using the loop refinement tool implemented in MODELLER (Fiser et al., 2000). Models were ranked according to the discrete optimized protein energy (DOPE) method (Shen and Sali, 2006), selecting the best-scoring loop out of 10 candidates. The same tool was used to reconstitute the backbone interruptions. Positions 491,495 and 812,816 were selected as anchors. The best-scoring loop was selected out of 10 candidates.

\section{Molecular Dynamics. System setup and parameters.}

Topologies to run coarse-grained (CG) simulations were generated with the martinize tool choosing the Martini v.2.1 force field with elastic network (de Jong et al., 2013; Monticelli et al 2008). The force bond constant was set to $500 \mathrm{~kJ} \mathrm{~mol}^{-1} \mathrm{~nm}^{-2}$ with lower and upper elastic bond cutoffs of 0.5 and $0.9 \mathrm{~nm}$ respectively. Firstly, 5000 steps of steepest descent with position restraints for the protein were run followed by 5000 steps without restraints. The obtained coordinates were inserted in a POPC:POPE (1:1) membrane via the insane tool (Wassenaar et al., 2015), where the membrane position was manually set up to match the reported transmembrane regions corresponding to residues 706-726 and 737-757 according to UniProt numbering (De Vecchis et al., 2017). All systems were fully solvated to mimic an environment of $150 \mathrm{mM}$ of $\mathrm{NaCl}$ solution. See Supplementary Table 1 for more details about each simulation setup.

All the final systems followed the same simulation protocol using the GROMACS 5.0.4 software (Abraham et al., 2015). Further 5000 steps of steepest descent minimisation with position restraints of $1000 \mathrm{~kJ} \mathrm{~mol}^{-1} \mathrm{~nm}^{-2}$ in protein and lipids were followed by 5000 steps without position restraints. Equilibration was performed in three stages, with timesteps of 20 fs. Firstly, 25000 steps of equilibration were run at $310 \mathrm{~K}$ using the V-rescale thermostat (Bussi et al., 2007) and semiisotropic pressure coupling via Berendsen barostat (Berendsen et al., 1984) with position restraints of $1000 \mathrm{~kJ} \mathrm{~mol}^{-1} \mathrm{~nm}^{-2}$ for protein and lipids, followed by the same setup without position restraints. Finally, the last equilibration step was run for 50000 steps with the V-rescale thermostat (coupling constant tau_t $=1$ ps) and semi-isotropic coupling with Parinello-Rahman barostat (Parinello and 
Rahman, 1981) (coupling constant tau $\_=12 \mathrm{ps}$ ). Production runs were $1 \mu$ s long for all the six systems, following the same parameters as those used in the last equilibration setup.

\section{Analysis.}

Root mean square deviations were calculated in GROMACS 5.0.4 via the gmx rms tool considering only backbone beads. Interfaces were obtained based on differences between solvent accessible surface areas obtained with the gmx sas tool, using a probe radius of $0.2638 \mathrm{~nm}$ in agreement with the $\mathrm{CG}$ water size. An interface between e.g protein $\mathrm{A}$ and $\mathrm{B}\left(\mathrm{I}_{\mathrm{A} / \mathrm{B}}\right)$ was calculated as the sum of the surface areas of protein $A$ and protein $B$ in the interface, following the equation: $I_{A / B}=I_{A}+I_{B}=$ $\left(\mathrm{SASA}_{\mathrm{A}}-\mathrm{SASA}_{\mathrm{A}_{-} \text {complex }}\right)+\left(\mathrm{SASA}_{\mathrm{B}}-\mathrm{SASA}_{\mathrm{B}_{-} \text {complex }}\right)$ where $\mathrm{SASA}_{\mathrm{A}}$ is the solvent accessible surface area calculated considering the protein $\mathrm{A}$ as isolated and $\mathrm{SASA}_{\mathrm{A}_{-} \text {complex }}$ is the solvent accessible surface area calculated for the protein A considering its solvent accessibility in the complex.

The distance in the membrane-normal $\mathrm{Z}$ axis between the phosphate beads from both bilayers (oriented in the $\mathrm{x}, \mathrm{y}$ plane) was measured in blocks (grid in $\mathrm{x}, \mathrm{y}$ of size $\sim 20 \AA$ ). $\mathrm{Z}$ coordinates of atoms corresponding to each block were averaged and subsequently, the distance between the average values of each block from the upper bilayer was subtracted from the lower one. Resulting difference inter-membrane distances between the final and initial structure of the production run are shown as a matrix.

\section{RESULTS AND DISCUSSION}

\section{Putative models for Fzo1 cis-dimers}

Mitochondrial tethering requires oligomerization of mitofusins in cis and then in trans (Anton et al., 2011; Fritz et al., 2001; Ishihara et al., 2004; Ishihara et al., 2003; Koshiba et al., 2004; Rapaport et al., 1998; Santel et al., 2003). We thus reasoned that focusing on cis-oligomerization aspects in first instance might facilitate investigating their general properties of assembly. In this regard, the Bacterial Dynamin Like Protein from Nostoc punctiforme represents a starting point of choice. BDLP was not only used as the template to generate our previous Fzo1 structural model (De Vecchis et al., 2017) but this bacterial DRP also displays well established cis-oligomerization properties (Low and Lowe, 2006; Low et al., 2009). Upon binding of non-hydrolysable analogues of GTP, BDLP operates an extensive conformational change from a compact structure (that was used as the template to generate the closed Fzo1 model, Fig. 1a) to an extended conformation. This extended conformation allows insertion of BDLP into lipid bilayers through a membrane paddle and favors its cis-oligomerization through GTPase domain interactions (PDB-id 2W6D, Low et al., 2009). The Fzo1 open-conformation model was obtained by superimposing our previously published closed conformation model piecewise onto the open structure of BDLP (Fig. 1b; see Methods for details). The final model of the Fzo1 dimer in open conformation was then superposed with respect to the template structure $2 \mathrm{~W} 6 \mathrm{D}$ using the $\mathrm{C} \alpha$ atoms (Fig. 1c). The resulting structural drift, measured as RMSD between target and template, is rather low at $0.63 \AA$, which underlines the similarity of both molecular systems. 


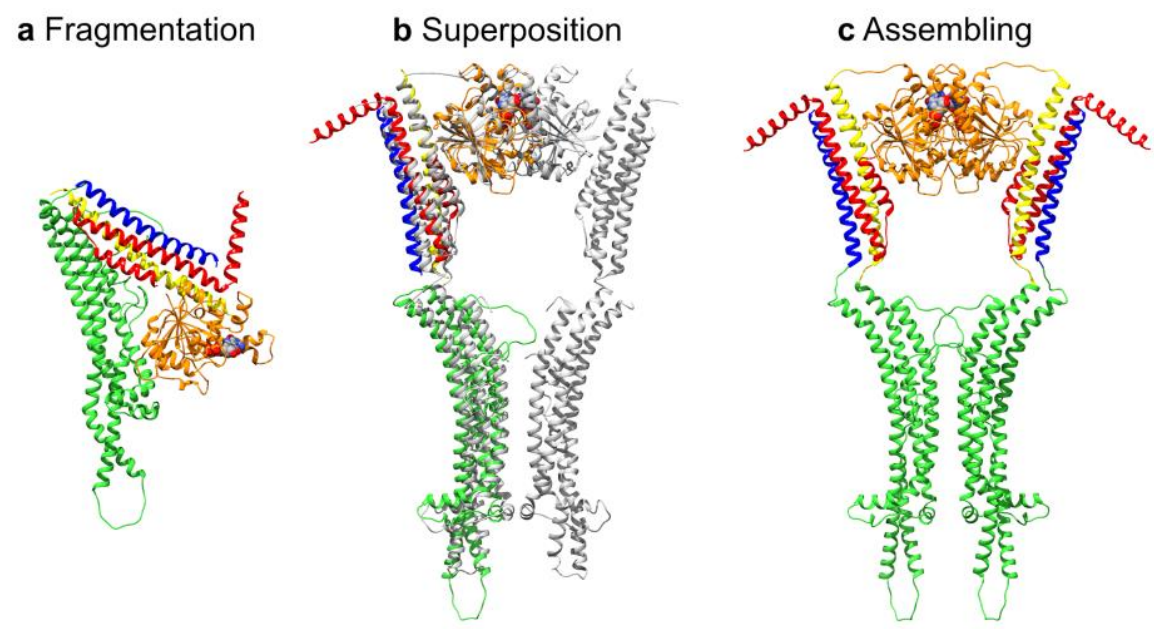

\begin{abstract}
Figure 1. Key steps of the modelling workflow to build a model of Fzo1 in open conformation, based on the BDLP structure. (a) The Fzo1 structural blocks delimited according to the putative hinges proposed for BDLP are highlighted by different colors. The blocks are coloured in rainbow from N-terminal (red) to C-terminal (blue). (b) Each block in Fzo1 (colored parts) was superposed to its corresponding fragment in $2 \mathrm{~W} 6 \mathrm{D}$ (gray ribbon). Note the backbone interruptions in the structure. (c) The final Fzo1 model in open conformation. Note that each GTPase domain (orange) from both chains is in close contact with each other. The GDP nucleotide is shown in space-filling representation.
\end{abstract}

The BDLP-like cis-oligomerization model (Fig. 2a) imposes an extensive conformational switch of Fzo1 with an opening angle of about $180^{\circ}$ between the four-helix bundle and the trunk of the mitofusin. While a possible conformational reorganization has been experimentally documented in this hinge region of Fzo1 (Cohen et al., 2011), a significantly lower angle of opening may not be excluded. GTPase domain contacts observed for human mitofusin (Cao et al., 2017) provide a template to form dimers with such a low opening angle (Fig. 2b). This assembly leads to a distinct head-to-head orientation with notably narrow Fzol opening that differs from the significantly wide Fzo1 opening seen in the BDLP-like cis-oligomerization model.

In either the wide or narrow head-to-head models, the GTPase domain interface favours cisdimerization of Fzo1 after GTP binding. This contrasts with the current view that oligomerization of mitofusins through their GTPase domain promotes their association in trans rather than cis (Cao et al., 2017; Yan et al., 2018). In this latter scenario, cis-oligomerization should thus employ Fzo1 interaction regions distinct from the GTPase domain. Interestingly, in our recent Fzo1 model, HR2 is exposed to the solvent suggesting it could be available for putative hydrophobic interactions with neighbouring Fzo1 molecules (De Vecchis et al., 2017). The coiled-coil structures would be positioned back-to-back to yield a cis-dimer with GTPase domains available for interactions in trans (Fig. 2c). This would notably be consistent with the crystal structure of the Mfn1 HR2 domain (Koshiba et al., 2004) that supports the possibility that mitofusins could also associate through their trunk region. However, in this crystal (PDB-id 1T3J), the interacting HR2 domains adopt an antiparallel-orientation suggesting that the transmembrane segments of two mitofusin molecules interacting through their trunks would be located on opposite membranes. 


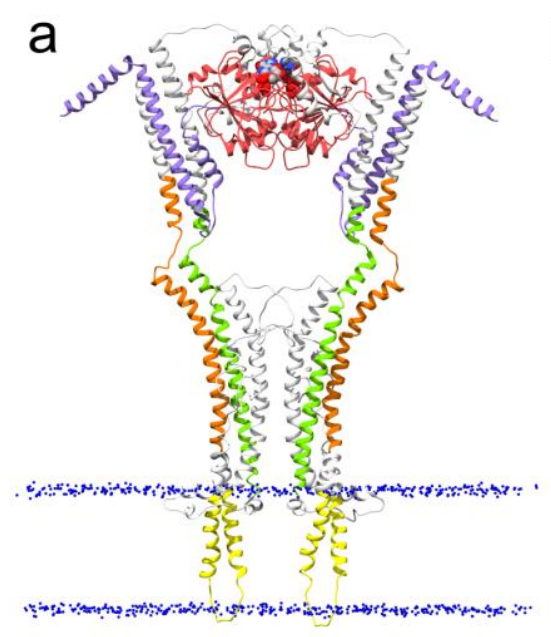

head-to-head cis interaction WIDE b

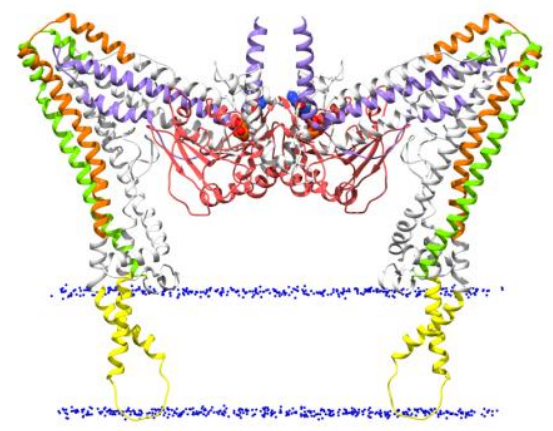

head-to-head cis interaction
C

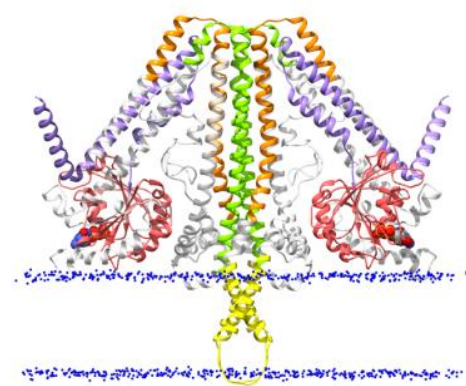

back-to-back cis interaction

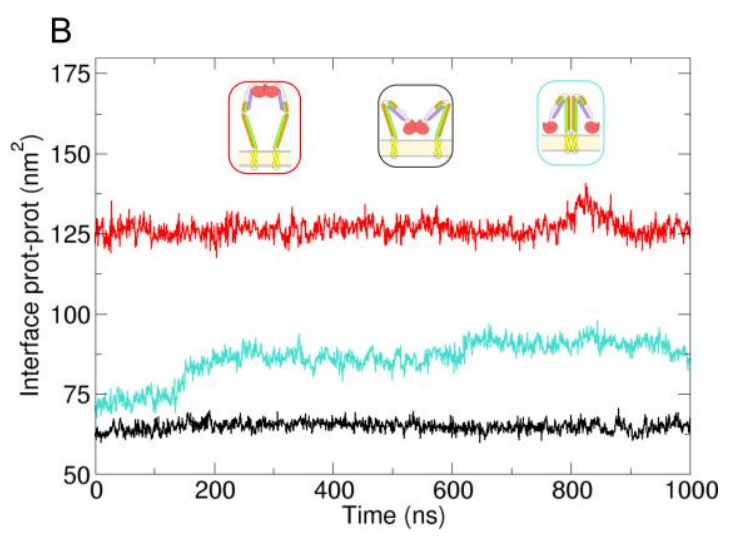

Figure 3. Lipid-protein and protein-protein interface evolution during CG MD simulations for the dimeric complexes. A Lipid-protein interfaces for the three different studied cis-dimers. B Protein-protein interfaces belonging to the three different cis-dimers. The colours in the curves match the outline of the corresponding image in the inset (black: head-to-head narrow, red: headto-head wide, cyan: back-to-back). The domains in the inset schemes are coloured in the same way as Figure 2.

We have tested the robustness of the three cis-dimer models through coarse-grained molecular dynamics (MD) simulations (see methods). The final conformations obtained for all three MD simulations stay close to their respective starting models (Supplementary Fig. 1). This observation suggests that the models are relatively stable, and therefore can all be considered as possible molecular assemblies (see detailed analysis in supplementary material). Notably, the dynamics of the closed back-to-back model shows a decrease in the membrane-protein interaction surface as 
well as an increase in the protein-protein interface (Fig. 3, cyan curve). The latter is a consequence of the augmented interaction of their transmembrane regions with each other that consequently decreases the lipid-protein interface. At this point ouf our study, MD simulations alone do not allow privileging the validity of any model over the others. For this reason we analyzed the propensity of each cis-dimer to possibly associate with the mitochondrial outer membrane carrier protein Ugo1 as cis-dimerization of Fzo1 has been suggested to involve the participation of Ugo1 (Anton et al., 2011). This 3-membrane spanning factor essential for outer membrane fusion (Coonrod et al., 2007; Hoppins et al., 2009; Sesaki and Jensen, 2001, 2004; Wong et al., 2003) interacts with Fzo1 through a region spanning residues 630-703 and 756-843 (Sesaki and Jensen, 2004) and may thus contribute deciphering the differential likelihood of the three cis-models. Interestingly, it is obvious that in the wide and narrow head-to-head dimers, the region required for potential interactions with Ugo1 is exposed and fully accessible (Fig. 4a, b). In contrast, this region becomes partially masked and likely less accessible to potential Ugo1 molecules in the back-toback model (Fig. 4c). If Ugo1 indeed participates in Fzo1 cis-dimers formation, these observations would thus tend to favour head-to-head models as opposed to the back-to-back configuration.

a

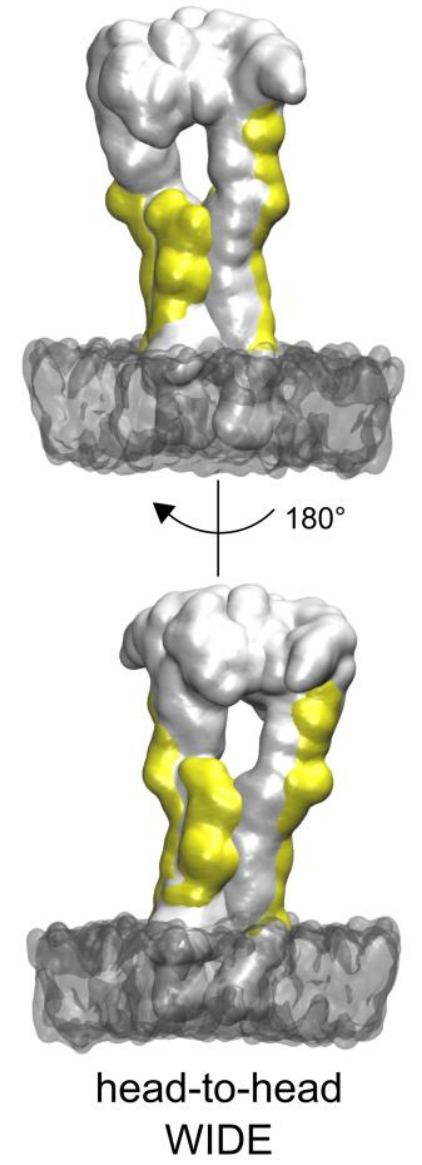

b
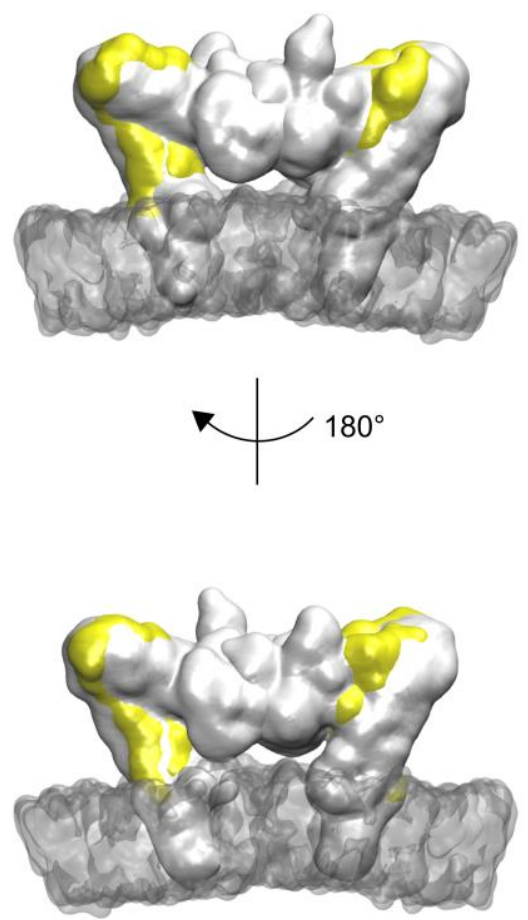

head-to-head
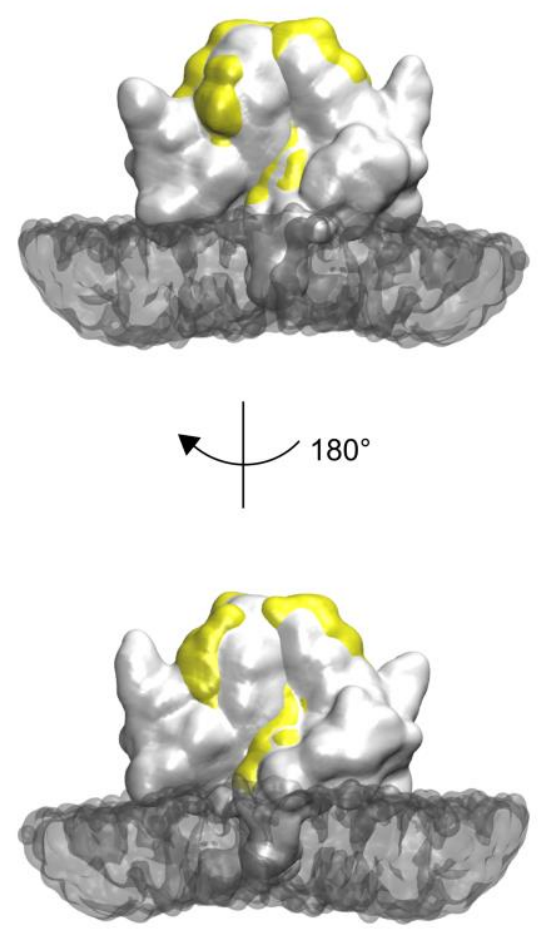

back-to-back

Figure 4. Exposed Ugo1-interacting regions in Fzo1 putative cis-dimers. a, b and c. Final snapshots from the simulations showing the Ugo1-interacting surfaces (yellow) for each of the cisdimers. Each Fzo1 monomer is represented with a different colour (magenta or cyan) and the membrane bilayer is depicted as a brown transparent surface.

\section{Proposed models for Fzo1 trans-tethered oligomers}

Based on the three cis-dimers of Fzo1 previously obtained, we aimed at modelling potential mitofusin trans-oligomers. We took here in consideration the possibility that cis-dimers from one membrane could engage in interactions with cis-dimers from an opposing membrane through the availability of either their GTPase or trunk domains. Importantly, the average $8 \mathrm{~nm}$ distance between outer membranes observed during Fzo1-mediated mitochondrial tethering (Brandt et al., 
2016) provides an experimental estimation of the membrane separation that Fzo1 trans-oligomers should favour.

Following GTP binding, wide opening of Fzo1 would take place and head-to-head cisdimerization through GTPase domains would generate competency for trans-oligomerization. In this configuration, trans interactions could take place through GTPase domains using an interface of association distinct from that used during cis-dimerization (Fig. 5a). Such a surface of interaction remains yet to be established experimentally. Moreover, the resulting Fzo1 trans-oligomer would impose a tethering distance of $32 \mathrm{~nm}$ between the two juxtaposed outer-membranes, which is four times higher than the average $8 \mathrm{~nm}$ distance experimentally observed (Brandt et al., 2016).

In the alternative configuration in which GTP binding would induce narrow opening of Fzo1, the most exposed surface of trans-interaction between head-to-head cis-dimers would lie in the trunk region. Anti-parallel associations reminiscent of those observed in the crystal structure of the Mfn1 HR2 domain (Koshiba et al., 2004) would take place between dimers from opposing membranes (Fig. 5c). This would impose a tethering distance of about $10 \mathrm{~nm}$, which is compatible with the cryo-ET experimental measures (Brandt et al., 2016).

In the context of the back-to-back HR-parallel cis-dimers (Fig. 2c) GTP binding would induce the conformational switch of Fzo1, which would allow the GTPase domain to engage in trans association with the GTPase domain of a back-to-back cis-dimer from an opposing membrane (Fig. 5b). Although this Fzo1 trans-oligomer imposes a tethering distance of $9 \mathrm{~nm}$ which is compatible with experimental observations, an extensive manipulation of the initial closed conformation involving hinges $1 \mathrm{a}$ and $1 \mathrm{~b}$ was required to generate the GTPase domain interface. Without this modification; the starting orientation of the GTPase domain would prevent the formation of the canonical G-interface observed in the dynamin superfamily (Daumke and Prafke, 2016) and Mfn1 dimers (Cao et al., 2017; Yan et al., 2018), (Supplementary Fig. 2).

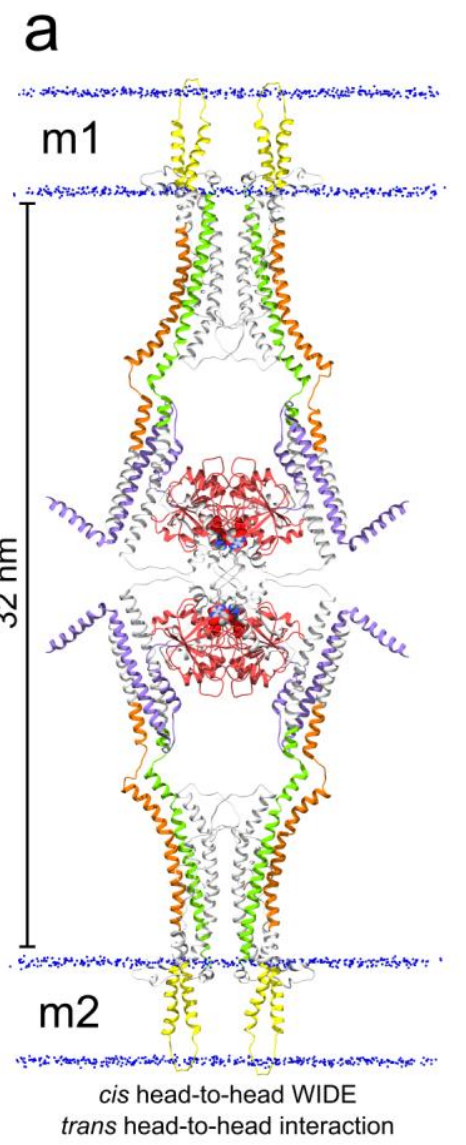

b

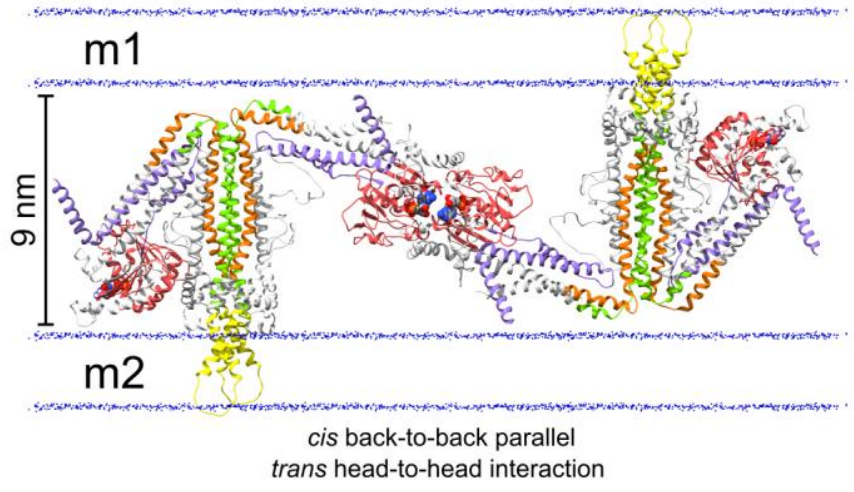

Figure 5. Putative model complexes for Fzo1 trans-tethering interactions. a, the Fzo1 models in

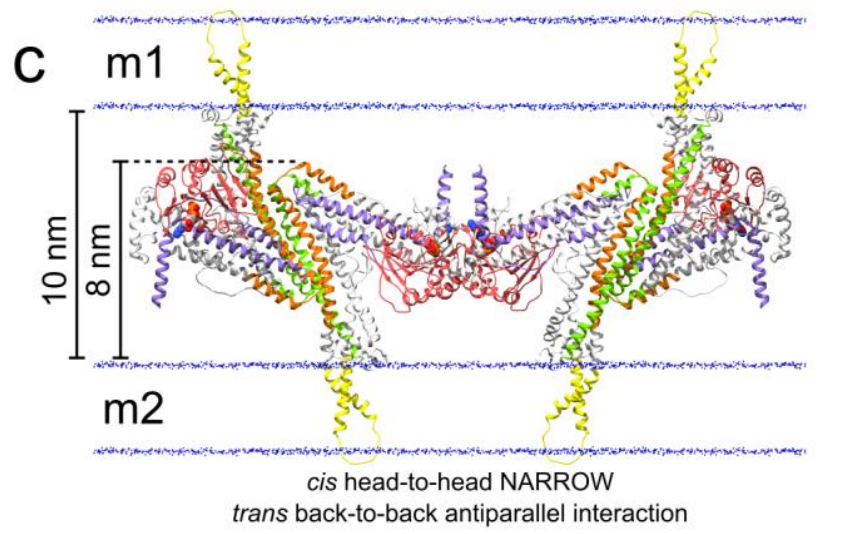
open conformations, as suggested from analogy to the BDLP system, interacting through their 
GTPase domains. b, the Fzo1 trans-tetramer in which the HRs are oriented in a parallel fashion and the trans interaction occurs towards the GTPase domain. c, the Fzo1 trans-tetramer in closed conformation. Here the interaction occurs towards the GTPase domain as well as through the respective HR domains oriented in an antiparallel fashion. Outer membranes corresponding to two different mitochondria are labelled as $\mathrm{m} 1$ and $\mathrm{m} 2$. The domains highlighted by color are: violet, HRN; green, HR1; orange, HR2; red, GTPase and yellow, transmembrane. Phosphorus atoms (blue) from lipid bilayer headgroups and GDP nucleotide are depicted in the space-filling representation.

\section{Trans-dimer dynamics.}

The robustness of the tetramers has been tested through coarse-grained molecular dynamics simulations. The final conformations obtained for all three MD simulations show a structural drift with respect to their respective starting models that, although significant (Supplementary Table 2), does not point to major issues in the structure (Fig. 6, 7). Thus, it is possible to infer that the models are stable in a membrane environment, and can therefore be considered plausible (see detailed analysis in supplementary material). When analysing the interface area between protein and lipids, we detected that the head-to-head model with parallel HR interactions has a higher interaction surface with the membrane compared to the other two tetramers (Fig 6a, red line). For this model, we have also observed a jump in the protein-protein interface in one of the two back-to-back cisdimers, as a consequence of an increased interaction between the transmembrane regions of each monomer (Fig 6c, red curve). Interestingly, the head-to-head trans antiparallel model showed an increase in membrane curvature during the simulation as a consequence of the strong interactions between the protein terminal regions and lipids as well as a region spanning residues 380-386 in the GTPase domain (Fig. 7c). Even taking in consideration that this model is missing the first 100 residues from the yeast mitofusin, the region including residues 380-386 appears to interact strongly in the back-to-back cis-dimer as well, where the terminals do not interact with the membrane. An analysis of the distance evolution between opposing membranes for all the tetrameric complexes showed that globally it changed slightly during the MD run without affecting the comparison with experiments. In particular, for the head-to-head trans anti-parallel interaction the initial distance between the membranes was $10 \mathrm{~nm}$, that value kept close only in the middle dimer $(9.5 \mathrm{~nm})$ after 1 $\mu \mathrm{s}$, while at the extremes of the dimer the intermembrane distance stabilised at approximately 5.5 $\mathrm{nm}$. This narrower value appeared as a consequence of the increased curvature in the membrane caused by the strong protein interactions with the lipid (Fig. 6c, Supplementary Fig. 3). 

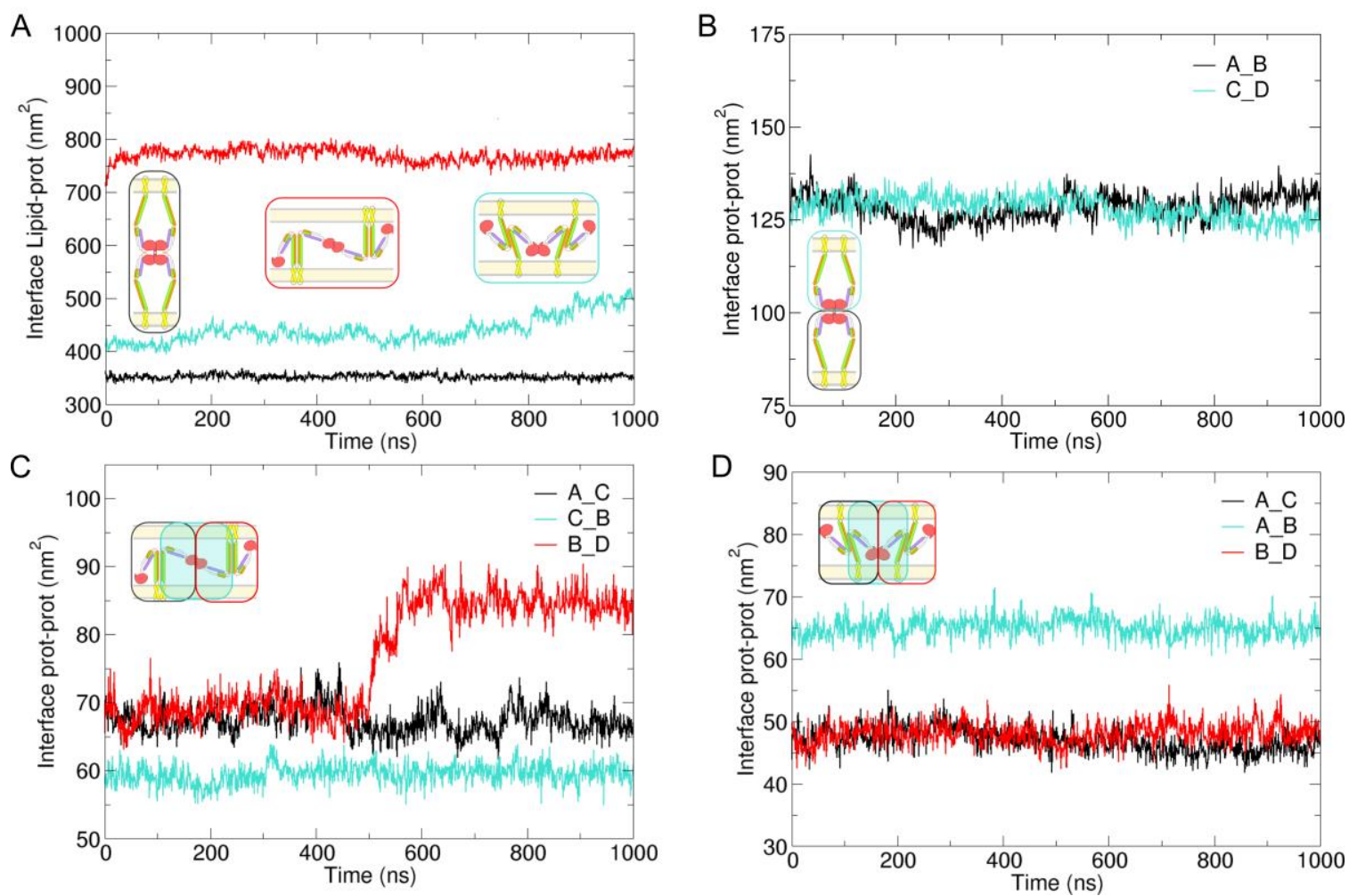

Figure 6. Lipid-protein and protein-protein interface evolution during CG MD simulations for the tetrameric complexes. A Lipid-protein interfaces for the three different studied tetrameric complexes (black: head-to-head trans open, red: head-to-head trans parallel, cyan: back-to-back trans antiparallel). B, C and $\mathbf{D}$ depict the evolution of the characteristic monomer-monomer interfaces belonging to the trans-tetramer in open conformation, the trans-tetramer in which the HRs are oriented in parallel and the trans-tetramer with HRs oriented in an antiparallel fashion. Legends A, B, C and D represent each of the monomers in the complex, following the coloring of image inset. 
a

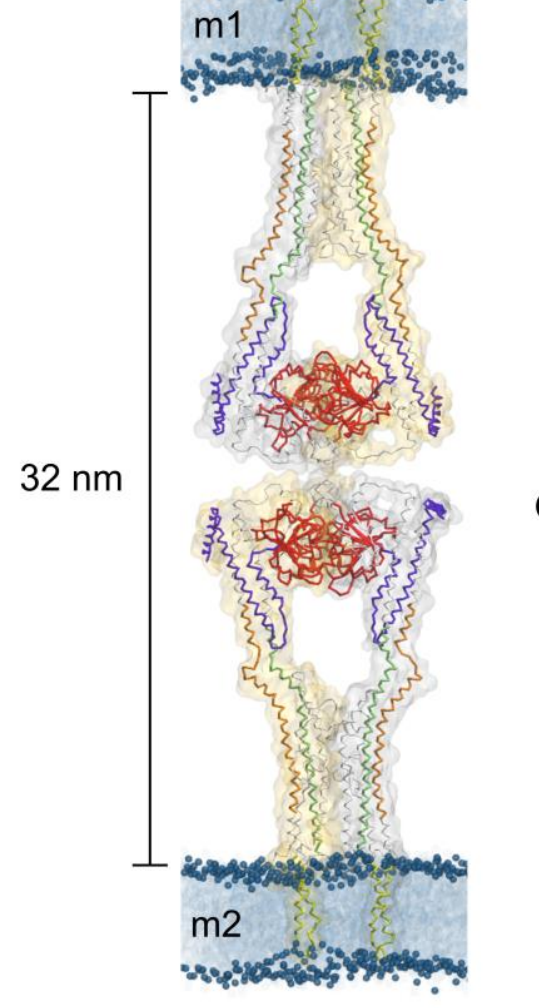

cis head-to-head WIDE

trans head-to-head interaction

C b

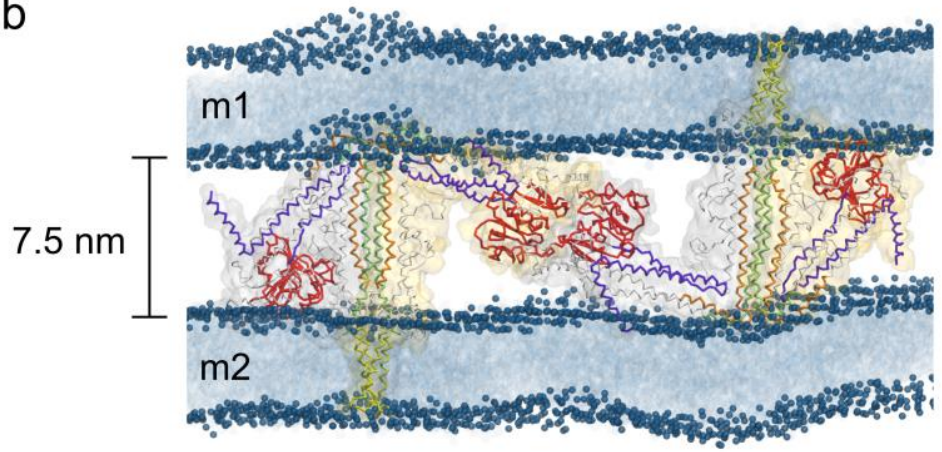

cis back-to-back parallel trans head-to-head interaction

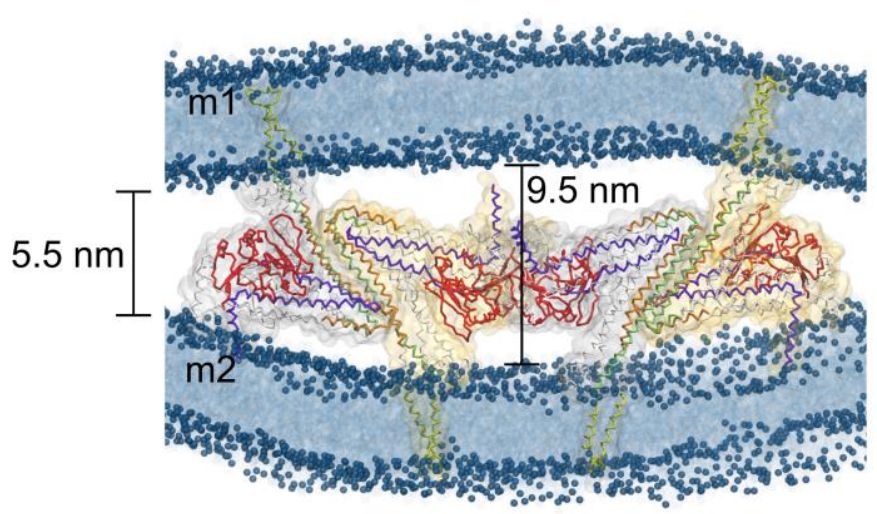

cis head-to-head NARROW

trans back-to-back antiparallel interaction

Figure 7. Final configurations of the putative model complexes for Fzo1 trans-tethering interactions after relaxation through molecular dynamics simulations. a, the Fzo1 models in open conformation, as suggested from the BDLP system analogy, interacting through their GTPase domains. b, the Fzo1 trans-tetramer in which the HRs are oriented in a parallel fashion and the trans interaction occurs towards the GTPase domain. c, the Fzo1 trans-tetramer in closed conformation. Here the interaction occurs towards the GTPase domain as well as through the respective HR domains oriented in an antiparallel fashion. In all figures the domains are colored: violet, HRN; green, HR1; orange, HR2; red, GTPase and yellow, transmembrane. Phosphorus atoms (dark blue) from the lipid headgroups of two mitochondria outer membranes $(\mathrm{m} 1, \mathrm{~m} 2)$ are depicted as spheres, whereas the tails are highlighted as a blue shadow. Intermembrane distances between the outer phosphate layer of the membranes are indicated.

\section{CONCLUSION}

In analogy to the macromolecular spirals formed during DRP-mediated membrane fission (Jimah and Hinshaw, 2019), DRP-mediated membrane fusion may require the formation of macrooligomers as observed with the Fzo1-dependent docking ring structure (Brandt et al., 2016). Such assemblies require building blocks as previously shown for fission DRPs that associate with their cofactors in the cytosol before recruitment to outer membranes and formation of the spirals (Koirala et al., 2013; Lackner et al., 2009; Mears et al., 2011). In the context of mitofusins, these building blocks would form on the same membrane imposing the assembly of cis-oligomers.

This rational led us to build 3 cis-assembly Fzo1 models that gave rise to 3 distinct transoligomeric models of mitofusin. Each model involves two main components of mitofusin autooligomerization: the GTPase domain interface (Cao et al., 2017; Yan et al., 2018) and the trunk domain interface (Koshiba et al., 2004). A plethora of experimental data demonstrates that the integrity of both regions is indeed essential for mitofusin-mediated mitochondrial fusion (Cohen et al., 2011; Eura et al., 2003; Griffin and Chan, 2006; Hermann et al., 1998; Honda et al., 2005; Koshiba et al., 2004; Rojo et al., 2002; Santel and Fuller, 2001). If considering BDLP, the GTPase 
domain interface should trigger oligomerisation in cis of Fzo1 (Low et al., 2009). Nonetheless, this interface is currently thought to trigger trans-oligomerization of mitofusins (Cao et al., 2017; Yan et al., 2018).

Only one out of our three final trans models employs this strategy. In the back-to-back cisdimers (with parallel-oriented HRs), two dimers from opposing membranes would engage in trans interactions through their GTPase domains (Fig. 2c, and 5b). Yet, the generation of this transtetramer required an extensive rotation of the GTPase domains around the axis of their four helix bundles to reconstitute the canonical G-interface where GTPase domains mirror each other in other members of the dynamin superfamily (Cao et al., 2017; Daumke and Praefcke, 2016). Moreover, a large area of the Ugo1 binding domain in each Fzo1 monomer was hindered by the HR interacting interface required to form the back-to-back cis-dimers (Fig. 4c). This model thus accumulates significant limitations upon confrontation to the experimental literature.

The two remaining models employ head-to-head cis-oligomerization properties. In this configuration, GTP bound Fzo1 would undergo opening that could be narrow to wide. As seen for the stabilization of GTP-bound fission DRPs with their cofactors (Lackner et al., 2009), this conformational switch around the hinges $1 \mathrm{a}$ and $1 \mathrm{~b}$ would be stabilized by concomitant binding of Ugo1 through the available trunk and a neighbouring mitofusin molecule through the activated GTPase domains. In both wide and narrow models the canonical G-interface is maintained. From then, wide or narrow cis-oligomers would engage in trans-association with Fzo1 cis-complexes from the opposing membrane.

The BDLP-like wide oligomers would associate in trans through a G-domain interface that is distinct from that employed during cis-dimerization. To our knowledge, such an interface has never been observed for other members of the dynamin superfamily. Moreover, these Fzo1 transoligomers would impose a tethering distance between outer membranes of $\sim 32 \mathrm{~nm}$, which is significantly larger than the distance observed experimentally between docked mitochondria $(\sim 8$ $\mathrm{nm}$, Brandt et al. 2016). Therefore, similar to the back-to-back system, the wide opening head-to head model is thus difficult to reconcile with the literature.

In contrast, the narrow opening head-to-head mechanism employs a trans-oligomerization strategy that turns out to fit particularly well with the past and current literature. The trunk region in the cis-oligomers would expose a hydrophobic spine from the HR2 that would be available for putative interactions (De Vecchis et al., 2017). These interactions would take place with an HR2 belonging to Fzo1 cis-oligomers from an opposing membrane in an anti-parallel fashion. This would be consistent with anti-parallel HR2 interactions observed in 2004 for Mfn1 (Koshiba et al., 2004). Moreover, the tethering distance of 5.5 to $9.5 \mathrm{~nm}$ imposed by this Fzo1 oligomerization model would agree with the $\sim 8 \mathrm{~nm}$ separation between outer membranes observed experimentally twelve years later (Brandt et al. 2016).

The narrow opening head-to-head cis-oligomerization followed by the antiparallel back-toback trans-associations system thus allows speculating on the possible outcome of the fusion complex. Following GTP hydrolysis, hinges $1 \mathrm{a}$ and $1 \mathrm{~b}$ might restore the displacement of the GTPase domain back to the trunk (i.e. a closed state). Similar to the dynamin BSE domain that may transfer energy from the GTPase domain to the trunk during DRP-mediated fission (Chappie et al., 2010; Daumke and Praefcke, 2016), this movement could induce a sliding of interacting HRs with respect to each other. This would contribute to further reducing the distance between outer membranes to $2-3 \mathrm{~nm}$ as previously observed (Brandt et al. 2016). This cycle would reiterate around this initial region of minimal contact to reach the docking stage characterized by an extended area of membrane apposition delimited by the ring-shaped mitochondrial docking complex (MDC).

It is tempting to propose that this MDC that stands in regions where the distance between outer membranes reaches 6 to $8 \mathrm{~nm}$ (Brandt et al. 2016) corresponds to a macromolecular assembly of Fzo1 oligomers reminiscent of those described in the present study. In addition, our models provide enough detail to derive experimentally testable hypotheses for future work, for instance in terms of possible crosslinks and surface-exposed residues. Conversely our models could be improved baased 
on experimental observations. Regardless of these possibilities we hope that our hypotheses will provide food for thoughts before elucidating the structure of full length mitofusins with their known or yet to be described cofactors as well as their precise oligomerization properties.

\section{BIBLIOGRAPHY}

Abraham, M.J., Murtola, T., Schulz, R., Páll, S., Smith, J.C., Hess, B., and Lindahl, E. (2015). GROMACS: High performance molecular simulations through multi-level parallelism from laptops to supercomputers. SoftwareX 1-2, 19-25.

Anton, F., Fres, J.M., Schauss, A., Pinson, B., Praefcke, G.J., Langer, T., and Escobar-Henriques, M. (2011). Ugo1 and Mdm30 act sequentially during Fzo1-mediated mitochondrial outer membrane fusion. J Cell Sci 124, 1126-1135.

Berendsen, H.J.C., Postma, J.P.M., van Gunsteren, W.F., DiNola, A., and Haak, J.R. (1984). Molecular dynamics with coupling to an external bath. J. Chem. Phys. 81, 3684-3690.

Bian, X., Klemm, R.W., Liu, T.Y., Zhang, M., Sun, S., Sui, X., Liu, X., Rapoport, T.A., and Hu, J. (2011). Structures of the atlastin GTPase provide insight into homotypic fusion of endoplasmic reticulum membranes. Proc Natl Acad Sci U S A 108, 3976-3981.

Brandt, T., Cavellini, L., Kuhlbrandt, W., and Cohen, M.M. (2016). A mitofusin-dependent docking ring complex triggers mitochondrial fusion in vitro. Elife 5 .

Bussi, G., Donadio, D., and Parrinello, M. (2007). Canonical sampling through velocity rescaling. J. Chem. Phys. 126, 14101.

Byrnes, L.J., Singh, A., Szeto, K., Benvin, N.M., O'Donnell, J.P., Zipfel, W.R., and Sondermann, H. (2013). Structural basis for conformational switching and GTP loading of the large G protein atlastin. Embo J 32, 369-384.

Cao, Y.L., Meng, S., Chen, Y., Feng, J.X., Gu, D.D., Yu, B., Li, Y.J., Yang, J.Y., Liao, S., Chan, D.C., et al. (2017). MFN1 structures reveal nucleotide-triggered dimerization critical for mitochondrial fusion. Nature 542, 372-376.

Cohen, M.M., Amiott, E.A., Day, A.R., Leboucher, G.P., Pryce, E.N., Glickman, M.H., McCaffery, J.M., Shaw, J.M., and Weissman, A.M. (2011). Sequential requirements for the GTPase domain of the mitofusin Fzo1 and the ubiquitin ligase SCFMdm30 in mitochondrial outer membrane fusion. J Cell Sci 124, 1403-1410.

Cohen, M.M., and Tareste, D. (2018). Recent insights into the structure and function of Mitofusins in mitochondrial fusion. F1000Res 7.

Coonrod, E.M., Karren, M.A., and Shaw, J.M. (2007). Ugo1p is a multipass transmembrane protein with a single carrier domain required for mitochondrial fusion. Traffic 8, 500-511.

De Vecchis, D., Cavellini, L., Baaden, M., Henin, J., Cohen, M.M., and Taly, A. (2017). A membrane-inserted structural model of the yeast mitofusin Fzo1. Sci Rep 7, 10217.

de Jong, D.H., Singh, G., Bennett, W.F.D., Arnarez, C., Wassenaar, T.A., Schäfer, L. V, Periole, X., Tieleman, D.P., and Marrink, S.J. (2013). Improved Parameters for the Martini Coarse-Grained Protein Force Field. J. Chem. Theory Comput. 9, 687-697.

Eura, Y., Ishihara, N., Yokota, S., and Mihara, K. (2003). Two mitofusin proteins, mammalian homologues of FZO, with distinct functions are both required for mitochondrial fusion. J Biochem 134, 333-344.

Fiser, A., Do, R.K., and Sali, A. (2000). Modeling of loops in protein structures. Protein Sci. 9, 1753-1773.

Friedman, J.R., and Nunnari, J. (2014). Mitochondrial form and function. Nature 505, 335-343.

Fritz, S., Rapaport, D., Klanner, E., Neupert, W., and Westermann, B. (2001). Connection of the mitochondrial outer and inner membranes by Fzo1 is critical for organellar fusion. J Cell Biol 152, 683-692.

Griffin, E.E., and Chan, D.C. (2006). Domain interactions within Fzo1 oligomers are essential for mitochondrial fusion. J Biol Chem 281, 16599-16606. 
Hermann, G.J., Thatcher, J.W., Mills, J.P., Hales, K.G., Fuller, M.T., Nunnari, J., and Shaw, J.M. (1998). Mitochondrial fusion in yeast requires the transmembrane GTPase Fzolp. J Cell Biol 143, 359-373.

Honda, S., Aihara, T., Hontani, M., Okubo, K., and Hirose, S. (2005). Mutational analysis of action of mitochondrial fusion factor mitofusin-2. J Cell Sci 118, 3153-3161.

Hoppins, S., Horner, J., Song, C., McCaffery, J.M., and Nunnari, J. (2009). Mitochondrial outer and inner membrane fusion requires a modified carrier protein. J Cell Biol 184, 569-581.

Monticelli, L., Kandasamy, S.K., Periole, X., Larson, R.G., Tieleman, D.P., and Marrink, S.-J. (2008). The MARTINI Coarse-Grained Force Field: Extension to Proteins. J. Chem. Theory Comput. 4, 819-834.

Ishihara, N., Eura, Y., and Mihara, K. (2004). Mitofusin 1 and 2 play distinct roles in mitochondrial fusion reactions via GTPase activity. J Cell Sci 117, 6535-6546.

Ishihara, N., Jofuku, A., Eura, Y., and Mihara, K. (2003). Regulation of mitochondrial morphology by membrane potential, and DRP1-dependent division and FZO1-dependent fusion reaction in mammalian cells. Biochem Biophys Res Commun 301, 891-898.

Jimah, J.R., and Hinshaw, J.E. (2019). Structural Insights into the Mechanism of Dynamin Superfamily Proteins. Trends Cell Biol 29, 257-273.

Koirala, S., Guo, Q., Kalia, R., Bui, H.T., Eckert, D.M., Frost, A., and Shaw, J.M. (2013). Interchangeable adaptors regulate mitochondrial dynamin assembly for membrane scission. Proc Natl Acad Sci U S A 110, E1342-1351.

Koshiba, T., Detmer, S.A., Kaiser, J.T., Chen, H., McCaffery, J.M., and Chan, D.C. (2004). Structural basis of mitochondrial tethering by mitofusin complexes. Science 305, 858-862.

Lackner, L.L., Horner, J.S., and Nunnari, J. (2009). Mechanistic analysis of a dynamin effector. Science 325, 874-877.

Low, H.H., and Lowe, J. (2006). A bacterial dynamin-like protein. Nature 444, 766-769.

Low, H.H., Sachse, C., Amos, L.A., and Lowe, J. (2009). Structure of a Bacterial Dynamin-like Protein Lipid Tube Provides a Mechanism For Assembly and Membrane Curving. Cell 139, 13421352.

Mears, J.A., Lackner, L.L., Fang, S., Ingerman, E., Nunnari, J., and Hinshaw, J.E. (2011). Conformational changes in Dnm1 support a contractile mechanism for mitochondrial fission. Nat Struct Mol Biol 18, 20-26.

Moss, T.J., Andreazza, C., Verma, A., Daga, A., and McNew, J.A. (2011). Membrane fusion by the GTPase atlastin requires a conserved C-terminal cytoplasmic tail and dimerization through the middle domain. Proc Natl Acad Sci U S A 108, 11133-11138.

Parrinello, M., and Rahman, A. (1981). Polymorphic transitions in single crystals: A new molecular dynamics method. J. Appl. Phys. 52, 7182-7190.

Pettersen, E.F., Goddard, T.D., Huang, C.C., Couch, G.S., Greenblatt, D.M., Meng, E.C., and Ferrin, T.E. (2004). UCSF Chimera--a visualization system for exploratory research and analysis. J. Comput. Chem. 25, 1605-1612.

Ramachandran, R. (2018). Mitochondrial dynamics: The dynamin superfamily and execution by collusion. Semin Cell Dev Biol 76, 201-212.

Rapaport, D., Brunner, M., Neupert, W., and Westermann, B. (1998). Fzolp is a mitochondrial outer membrane protein essential for the biogenesis of functional mitochondria in Saccharomyces cerevisiae. J Biol Chem 273, 20150-20155.

Rojo, M., Legros, F., Chateau, D., and Lombes, A. (2002). Membrane topology and mitochondrial targeting of mitofusins, ubiquitous mammalian homologs of the transmembrane GTPase Fzo. J Cell Sci 115, 1663-1674.

Santel, A., Frank, S., Gaume, B., Herrler, M., Youle, R.J., and Fuller, M.T. (2003). Mitofusin-1 protein is a generally expressed mediator of mitochondrial fusion in mammalian cells. J Cell Sci 116, 2763-2774.

Santel, A., and Fuller, M.T. (2001). Control of mitochondrial morphology by a human mitofusin. J Cell Sci 114, 867-874. 
Sesaki, H., and Jensen, R.E. (2001). UGO1 encodes an outer membrane protein required for mitochondrial fusion. J Cell Biol 152, 1123-1134.

Sesaki, H., and Jensen, R.E. (2004). Ugo1p links the Fzo1p and Mgm1p GTPases for mitochondrial fusion. J Biol Chem 279, 28298-28303.

Shen, M.-Y., and Sali, A. (2006). Statistical potential for assessment and prediction of protein structures. Protein Sci. 15, 2507-2524.

Shutt, T.E., and McBride, H.M. (2013). Staying cool in difficult times: mitochondrial dynamics, quality control and the stress response. Biochim Biophys Acta 1833, 417-424.

Tilokani, L., Nagashima, S., Paupe, V., and Prudent, J. (2018). Mitochondrial dynamics: overview of molecular mechanisms. Essays Biochem 62, 341-360.

Westermann, B. (2010). Mitochondrial fusion and fission in cell life and death. Nat Rev Mol Cell Biol 11,872-884.

Wong, E.D., Wagner, J.A., Scott, S.V., Okreglak, V., Holewinske, T.J., Cassidy-Stone, A., and Nunnari, J. (2003). The intramitochondrial dynamin-related GTPase, Mgm1p, is a component of a protein complex that mediates mitochondrial fusion. J Cell Biol 160, 303-311. basis for GTP hydrolysis and conformational change of MFN1 in mediating membrane fusion. Nat Struct Mol Biol 25, 233-243. Cell Biol 210, 961-972.

\section{ACKNOWLEDGEMENTS}

Funding for this work was provided by the French Agency for Research and "Initiative d'Excellence" (ANR-11-LABX-0011, cluster of excellence LABEX Dynamo). This work was performed using HPC resources from GENCI-CINES (grant number 2016-072292). Research in the Cohen laboratory is supported by the ANR grant MOMIT (ANR-17-CE13-0026-01). 
Supplementary:

Supplementary Table 1: Molecular dynamics simulation details for each system

\begin{tabular}{|c|c|c|c|}
\cline { 2 - 4 } \multicolumn{2}{c|}{} & Box size $(\mathrm{x}, \mathrm{y}, \mathrm{z}[\mathrm{nm}])$ & \multicolumn{2}{c|}{ Number of particles } \\
\cline { 2 - 4 } & \multicolumn{2}{|c|}{ All } & Protein + lipid \\
\hline Head-to-head wide & $25.1474 \times 25.1474 \times 28.9065$ & 157017 & 27418 \\
\hline Head-to-head narrow & $24.6357 \times 24.6357 \times 29.8118$ & 156752 & 27058 \\
\hline Back-to-back & $25.2349 \times 25.2349 \times 28.7671$ & 157232 & 27298 \\
\hline Head-to-head trans & Tetramers & & \multicolumn{2}{|c|}{78836} \\
\hline Head-to-head trans parallel & $29.4832 \times 29.4832 \times 67.4888$ & 523921 & 166292 \\
\hline Head-to-head trans antiparallel & $30.4625 \times 40.6166 \times 32.2698$ & 347636 & 101324 \\
\hline
\end{tabular}

618

619

\section{a}

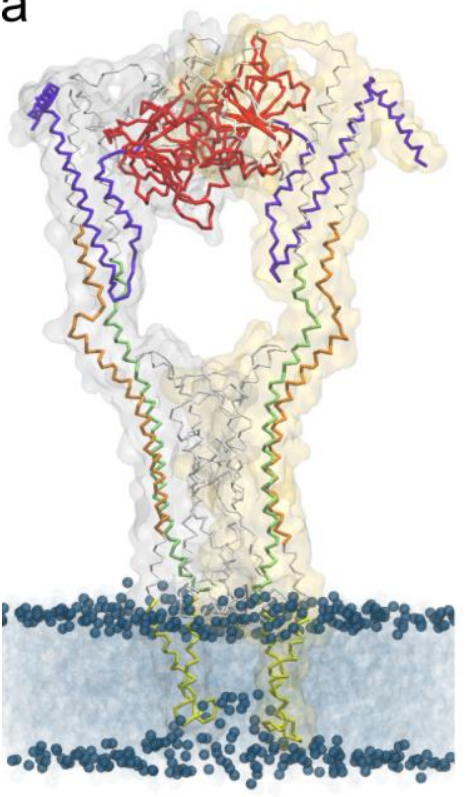

head-to-head

cis interaction

WIDE b

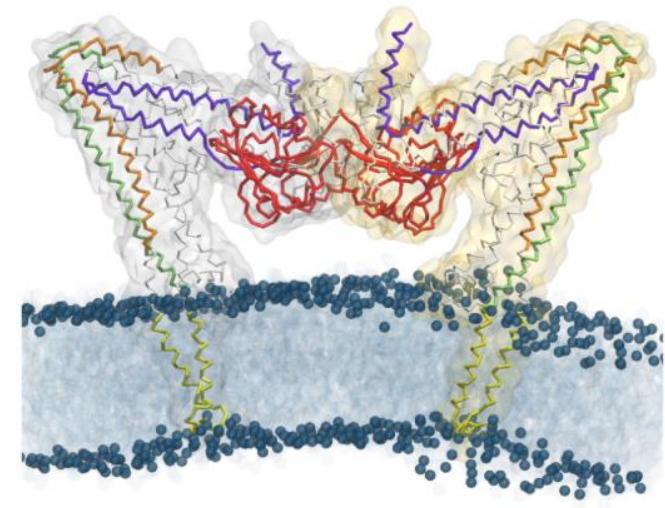

head-to-head

cis interaction

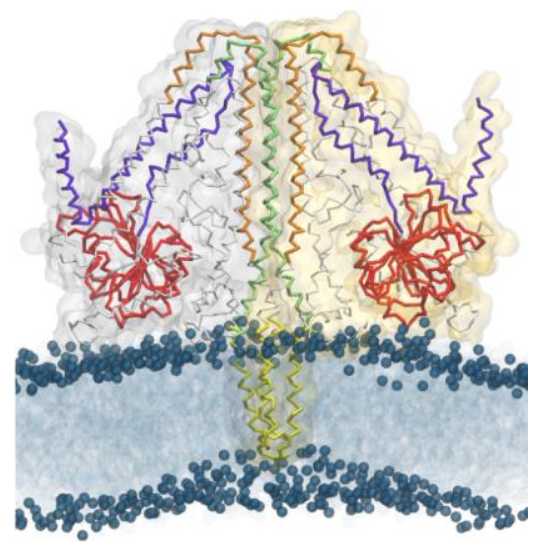

back-to-back cis interaction
620

621

622

623

624

625

626

627

628

629

630
C

Supplementary Figure 1: Putative membrane-inserted Fzo1 models interacting in cis configuration after molecular dynamics relaxation. a and b. Wide and narrow head-to-head complexes, respectively. c. Back-to-back complex of the closed model. The domains are colored: violet, HRN; green, HR1; orange, HR2; red, GTPase and yellow, transmembrane. Phosphorus atoms (dark blue) from the lipid bilayer headgroups are depicted as spheres, whereas the bilayer is highlighted as a blue shadow.

Supplementary Table 2: RMSD values averaged over the last 100 ns of simulation for each dimer (Figure 2a, b, c) and tetramers (Figure 3a, b, c). Reported values include a full RMSD calculated over all the backbone particles (BB) as well as by every chain and structural fragment separately. 


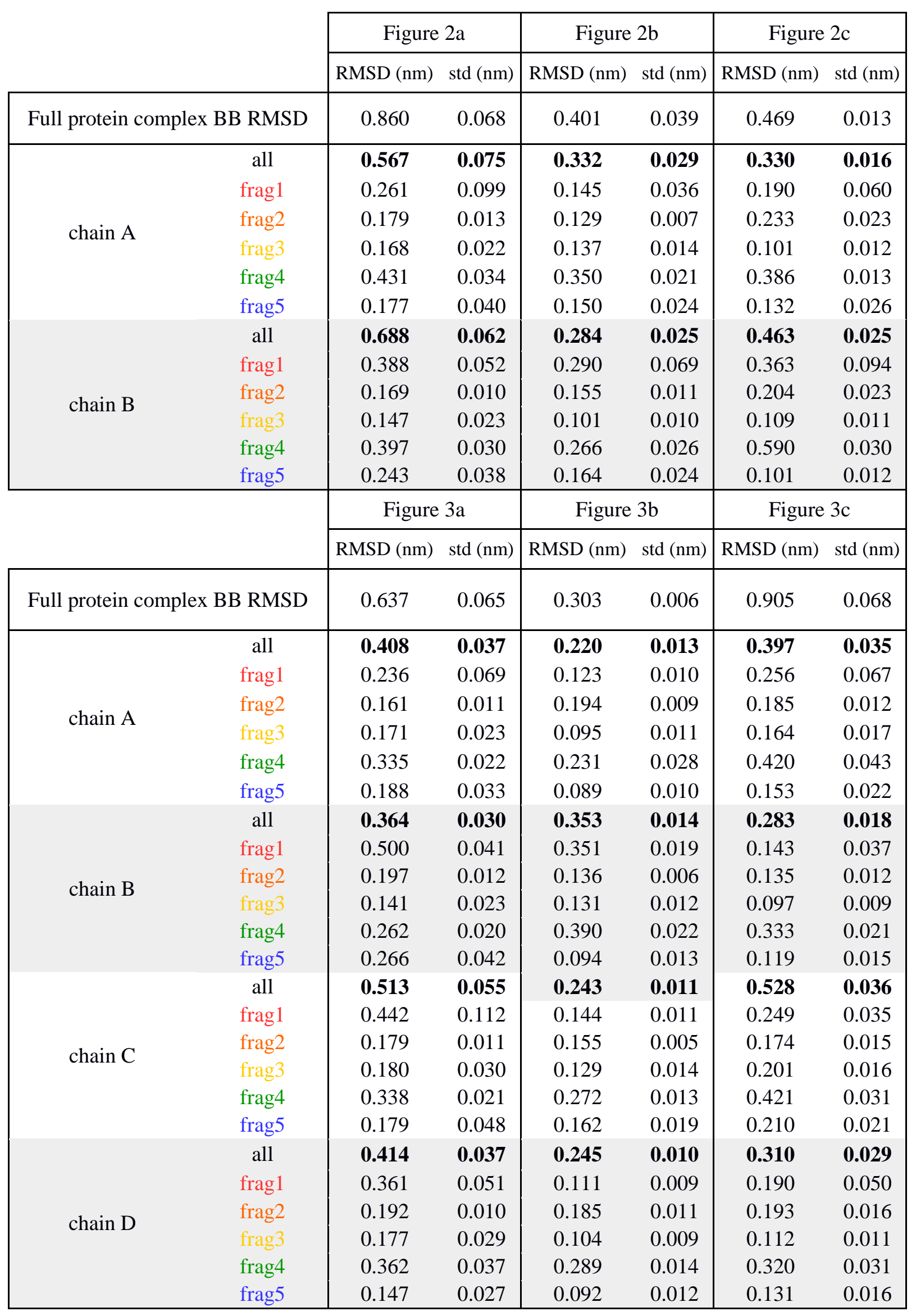


a

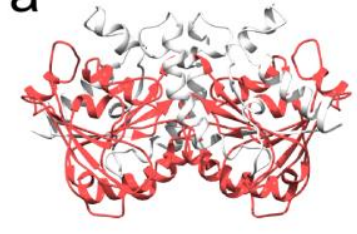

b

Supplementary Figure 2. a. The Fzo1 GTPase dimer construct in which the canonical G-interface commonly observed in the dynamin superfamily (Daumke and Prafke, 2016) is reconstituted. b. The back-to-back HR-parallel trans-tetramer during the modeling procedure (see Method). The Figure shows how the two GTPase domains that interact in trans share an apparent incorrect orientation (compared with a). Without an extensive conformational rearrangement that would involve both hinges $1 \mathrm{a}$ and $1 \mathrm{~b}$, the formation of the canonical G-interface observed in the dynamin superfamily (Daumke and Prafke, 2016) would be impeded. In particular, the dotted line should be located on the same side. The domains are: violet, HRN; green, HR1; orange, HR2; red, GTPase and yellow, transmembrane. Phosphorus atoms (blue) from lipid bilayer headgroups and GDP nucleotide are depicted in the space-filled representation.
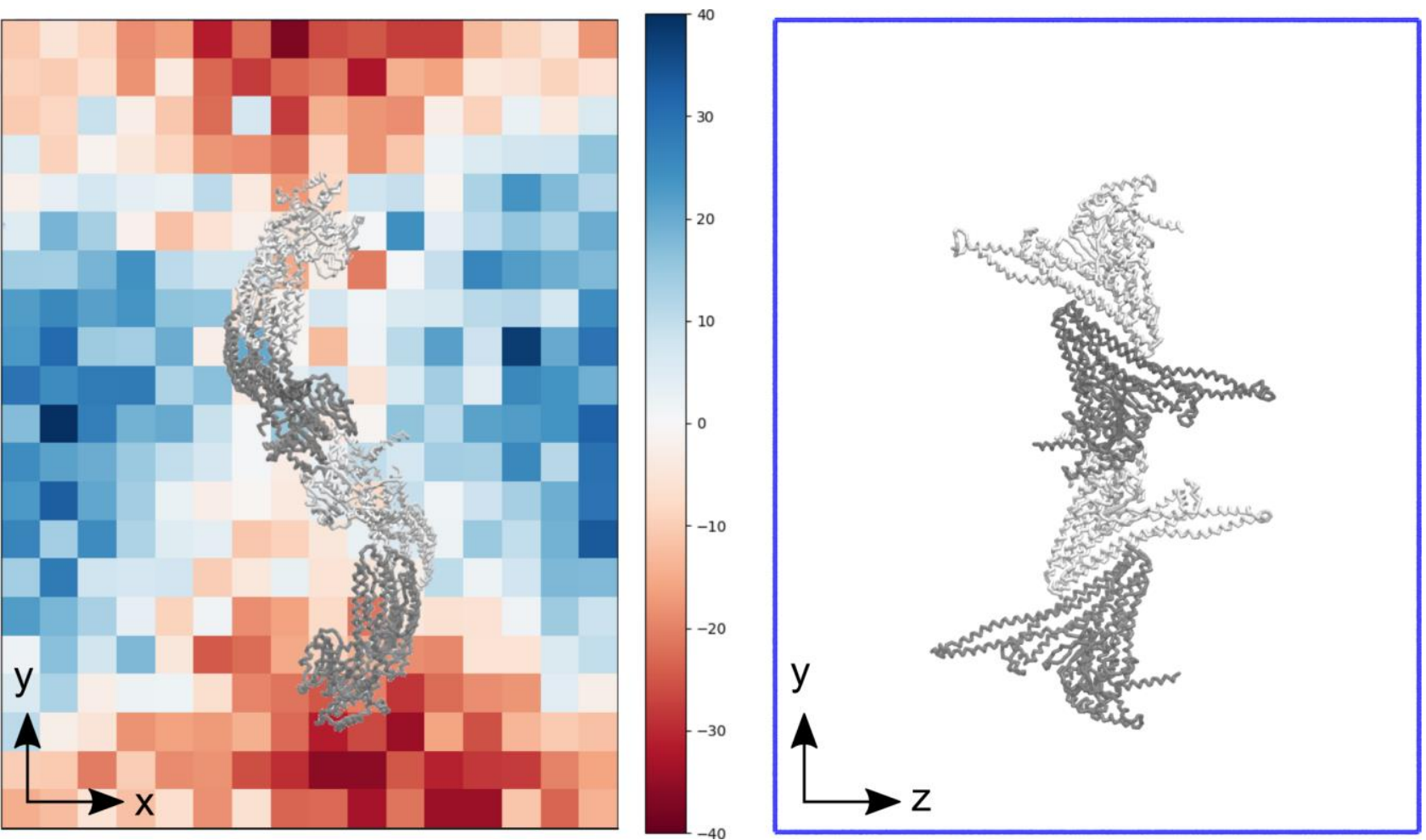

Supplementary Figure 3: Inter-bilayer difference distance matrix $(\AA)$. Left panel shows the final difference in distances compared to the initial structure, red regions correspond to regions that 
651 got closer in space compared to the initial structure of the production run whereas blue regions 652 represent areas that moved further apart. The right panel shows a rotation of 90 degrees around the $653 \mathrm{y}$-axis to visualize the position of the complex. 